\title{
Instantaneous, three-component planar Doppler velocimetry using imaging fibre bundles
}

\author{
D.S. Nobes, H.D. Ford, R.P. Tatam
}

\begin{abstract}
This paper describes a planar Doppler velocimetry (PDV) technique that is capable of measuring the three, instantaneous and time average components of velocity over two spatial dimensions using a single pair of signal and reference cameras. The three views required to obtain three-component velocity information are guided from the collection optics to a single imaging plane using flexible fibre imaging bundles. These are made up of a coherent array of single fibres and are combined at one end as the input plane to the measurement head. Measurements of the velocity field of a rotating disk are used in the development of the technique and initial results of the instantaneous velocity field of a jet are presented.
\end{abstract}

\section{1}

\section{Introduction}

Detailed information of flow properties and flow phenomena is an essential part of engineering design of flow systems and the development of the theory of fluid mechanics. With this in mind and with the advent of suitable hardware, new techniques to measure and investigate flows have been developed. One of these is planar Doppler velocimetry (PDV) (Samimy and Wernet 2000), which is also known in the literature as Doppler global velocimetry (DGV) (Meyers and Komine 1991).

General reviews of the development of PDV have highlighted two themes in the development of the technique (Samimy and Wernet 2000; Mosedale et al. 2000; Elliot and Beutner 1999; Reinath 1997). The first is the development of systems for three-component velocity measurements over a plane using two-dimensional array cameras. The high cost of these devices has pushed researchers to minimise the number used in the technique. The second focuses on ongoing problems in the development of PDV on system stability and especially the behaviour of the light source used. The latest

Received: 11 October 2002 / Accepted: 13 January 2003

Published online: 26 March 2003

(C) Springer-Verlag 2003

D.S. Nobes ( $₫)$, H.D. Ford, R.P. Tatam Optical Sensors Group, School of Engineering, Cranfield University, Cranfield, Bedfordshire, MK43 0AL, UK

E-mail: d.nobes@cranfield.ac.uk

Tel.: +44-1234-750111

Fax: +44-1234-752452

The work was funded by Engineering and Physical Research Sciences (EPSRC) UK, and The Royal Society (UK). implementation of ideas into PDV and some of the latest applications are reported in a special issue of the journal Measurement Science and Technology (Tropea 2001). Building on this work, this paper describes the development of a novel PDV system that addresses these two themes.

2

\section{Background}

The theory of PDV is based on the Doppler principle that light, when scattered from a moving object is frequency shifted depending on the velocity of that object. The Doppler formula describes the relationship between the velocity of the object and the frequency shift of the light scattered from that object and can be expressed using the vector equation

$\Delta v=\frac{v_{\mathrm{o}}}{c}(\mathbf{o}-\mathbf{i}) \mathbf{V}$

Here, $\Delta v=v_{\mathrm{D}}-v_{\mathrm{o}}$ is the difference between the Doppler shifted frequency $\left(v_{\mathrm{D}}\right)$ and the frequency of the light source $\left(v_{\mathrm{o}}\right), c$ is the free space speed of light, $\mathrm{V}$ is the velocity vector of the object that is scattering the light and $\mathbf{o}$ is a unit direction vector to the viewer and $\mathbf{i}$, the unit direction vector of the light source. A physical interpretation of this equation can be developed for the threedimensional case when the light source and the viewing direction are not in the same plane, as shown in Fig. 1.

From Eq. (1) it can be seen that to determine the velocity of the scattering object, the Doppler shift of the scattered laser light needs to be determined as well as having knowledge of the propagation direction of the light source and the direction from which the scattered signal is collected. While the light source and viewing direction can be easily determined, a method for determining the Doppler shift of the light frequency is needed. The PDV technique measures the shift in frequency directly using a frequency-to-intensity converter. Essentially, a filter that attenuates the scattered signal based on the frequency of that signal. For this filter to be efficient, it must have a cutoff that extends over the maximum expected range of velocities. For this a basic example of the expected performance of the filter can be developed. If a laser at a wavelength of $532 \mathrm{~nm}$ is used as the light source and the minimum to maximum expected velocity range is $\pm 100 \mathrm{~m} /$ $\mathrm{s}$ then the Doppler shift will be $\pm 200 \mathrm{MHz}$ depending on o and $\mathbf{i}$. In this example the filter will then need to have a maximum cut-off for just over $400 \mathrm{MHz}$.

For such a narrow operation range Komine (1990) identified the use of the atomic and molecular absorption bands. These absorption bands are spectroscopic lines and 


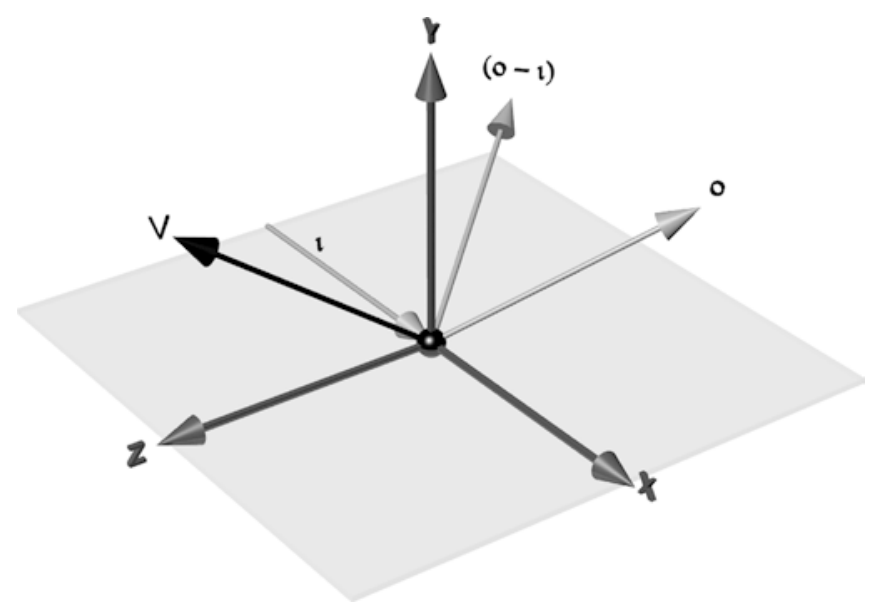

Fig. 1. The three-dimensional Doppler. A schematic of the Doppler principle: $\mathrm{V}$ vector of the scattering particle; $\mathrm{i}$ light illumination vector; o observation vector; $(\mathbf{o}-\mathbf{i})$ sensitivity vector

are at specific frequencies that equate to the energy transfer levels of the substance used in the filter. Several substances have been identified that have suitable properties that could potentially be utilised (Miles et al. 2001; Chan et al. 1995). Of these substances, iodine has an absorption band in the narrow tuning range of commercial argon-ion lasers and several absorption bands in the larger tuning range of frequency doubled Nd:YAG lasers (Miles et al. 2001).

The philosophy of the PDV technique is to tune a narrow line-width laser light source to a point that is half way either up or down the side of an absorption (Meyers and Komine 1991). For no shift in the laser frequency, or zero velocity measured, the detector will see a signal of $50 \%$ of the value of full transmission. Any Doppler shift in the laser frequency will be measured as a change in the signal intensity level. The sign of the velocity direction of the measured component can also be determined from the direction of the shift to either lower or higher intensities.

Early in the development of PDV it was realised that to obtain usable data a reference signal was needed (Komine 1990). The reference signal splits off before the filter is used to normalise the signal measured through the filter, correcting for variations in the scattered intensity. There are two sources that vary the intensity of the scattered signal. The power of the light source can vary in time as well as the scattering efficiency of the scattering medium. For measurement of flow velocities, a laser can be spread into a light sheet. The power distribution in this sheet can vary spatially across the sheet and in time. The flow is seeded with particles that are designed to follow the flow (Meyers 1991). The amount of seeding and the size of the particles affect the scattering efficiency and hence the intensity of the scattered signal. Hence, by normalising the signal with a reference, the effect of intensity variation caused by laser power fluctuations and seeding density can be removed.

Other than the work of Roehle and coworkers (Roehle 1996; Roehle et al. 2000) all other PDV systems found by the authors in the literature follow the general scheme of a single laser source and multiple viewing directions. Initial work used CW lasers (Komine 1990; Ford and Tatam 1997). However, many research groups quickly moved to pulsed, frequency doubled Nd:YAG systems (Reinath 1997; Elliot and Beutner 1999; Nobes et al. 2002) with the main aim of increasing the available laser power while at the same time making instantaneous velocity measurements possible. Meyers and Komine (1991) began the initial work in the area using a CW argon-ion laser and their technique is perhaps the classic arrangement of PDV. Here, six cameras in three pairs of signal and reference cameras viewed a region of interest from three different directions, each pair utilising its own iodine cell. This style of arrangement of the two cameras is shown in Fig. 2a. Some of the reported difficulties with this system included the need to develop frame grabbers that could sample from two cameras at once (not commercially available at the time), integration of a network of computers to control and drive the equipment, low signal to noise from the interlaced frame cameras used, differential sensitivity of the different iodine cells and the laser to vibration and temperature and the extensive development of software for control of the experiment as well as the processing of data.

The introduction of digital technology has seen the use of CCD cameras and commercial frame grabbers that support multiple cameras. Cooled CCD cameras can also a

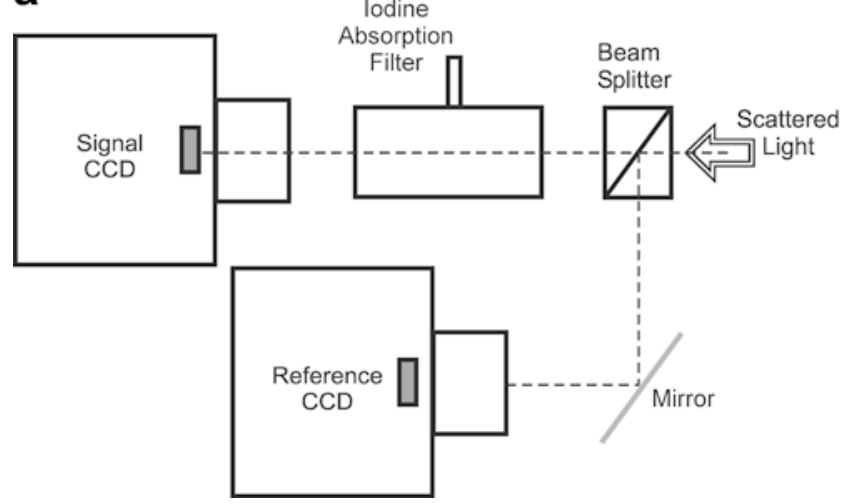

Fig. 2a, b. Two camera arrangments. a The arrangement of equipment used in a standard PDV head and $\mathbf{b}$ the arrangement used in a split view PDV system

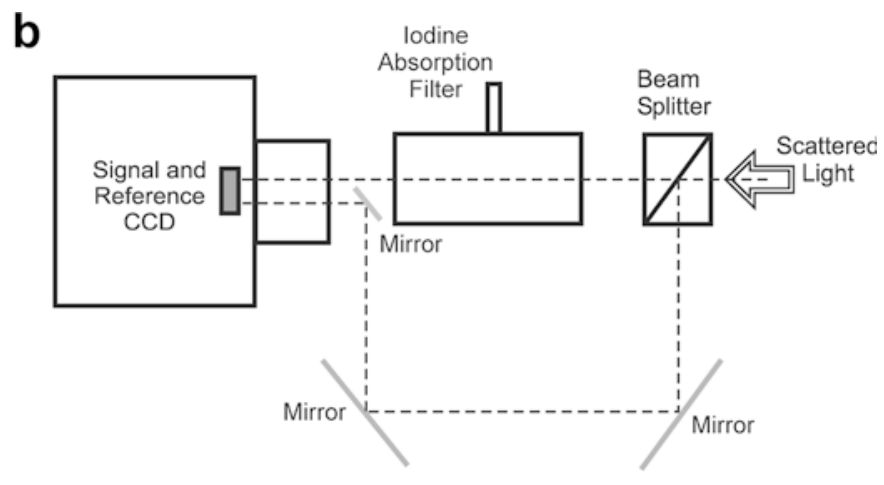


provide high signal to noise as well as higher data depths up to 16 bit (Meyers et al. 1998). These are expensive however, which has triggered the development of a number of novel arrangements to reduce the number of cameras used. One alternative has focussed on using a single camera to detect both the signal and reference images (Smith et al. 1996). A typical arrangement of the split view technique is shown Fig. 2b. Viewing from three directions, this reduces the number of cameras from six to three at the expense of lower spatial resolution. Another alternative is to use a duel frame camera and two consecutive laser pulses of different frequency to capture a signal image with the laser tuned onto the transfer function and reference image with the laser shifted off the transfer function. In this arrangement, three sets of a single camera and iodine cell would be required and this arrangement would guarantee alignment between the signal and reference images.

For any of the layouts of the detector equipment, frequency drift of the laser is an important problem, especially for pulsed Nd:YAG systems. For these lasers an injection-seeded laser is used to tune the host laser. The seed laser has a narrow line width $(\sim 5 \mathrm{kHz}) \mathrm{CW}$ laser at the fundamental frequency of the host laser $(1064 \mathrm{~nm})$. This seed laser, which is frequency tuneable by changing the temperature of the cavity crystal, is introduced into the cavity of the host laser and because its power is orders of magnitude larger than the noise in the system, the host laser builds up preferentially around this frequency. To lock the host laser to the seed laser the length of the cavity of the host laser is adjusted. This is done in a feedback manner with the position of one of the mirrors of the host being dithered. This results in an output that can be tuned over the gain curve of the host laser by tuning the seed laser and dithers about the frequency of the seed laser. The amount of dither is dependent on the set-up of the whole laser system but can be of the order of $\pm 50 \mathrm{MHz}$. To counter this movement of the frequency of the host laser many research groups have employed a system to measure the frequency of the laser for the pulse that is used to take the measurement. A typical system is an arrangement of single point detectors and a dedicated iodine cell (Mosedale et al. 2000; Elliot and Beutner 1999).

\section{3}

\section{Experimental set-up}

The PDV system described here has been designed to address several of the problems and limitations of PDV systems that have been reported in the literature. Overall, the following criteria have focused the structure of the current PDV system. The PDV system will aim to (1) measure the three components of velocity over a plane, (2) be capable of both instantaneous as well as time-average measurements, (3) measure internal as well as external flows, (4) minimise the number of cameras used, (5) address problems of laser stability and (6) address problems of stability of the iodine cell.

To achieve these aims a PDV system has been developed that uses the philosophy of one illumination direction and three viewing detectors. A schematic of the experimental arrangement is shown in Fig. 3. To achieve the aim of instantaneous measurement of velocity a pulsed frequency doubled Nd:YAG laser is used (Spectra Physics GCR 190-30). This laser is capable of producing $10 \mathrm{~ns}$ pulses with $300 \mathrm{~mJ}$ at $532 \mathrm{~nm}$. A second CW laser (Lightwave Model 101) is coupled to and seeds the host laser at the host laser fundamental frequency of $1064 \mathrm{~nm}$. The fundamental output from the host laser is frequency doubled to $532 \mathrm{~nm}$ using a second harmonic generator and this is used as the light source for the experiment. The laser pulse is spread into a light sheet using a combination of spherical and cylindrical lenses.

A unique feature of this PDV system is that a single head is utilised consisting of two cooled CCD cameras (LaVision Imager3) and a single iodine cell. The cameras operate at $15^{\circ} \mathrm{C}$ giving low noise performance. The arrays are $1,280 \times 1,024$ made up of square $6.7 \mu \mathrm{m}$ pixels and images collected are digitised to $12 \mathrm{bit}$. A single iodine cell is enclosed in a heated oven with the operation

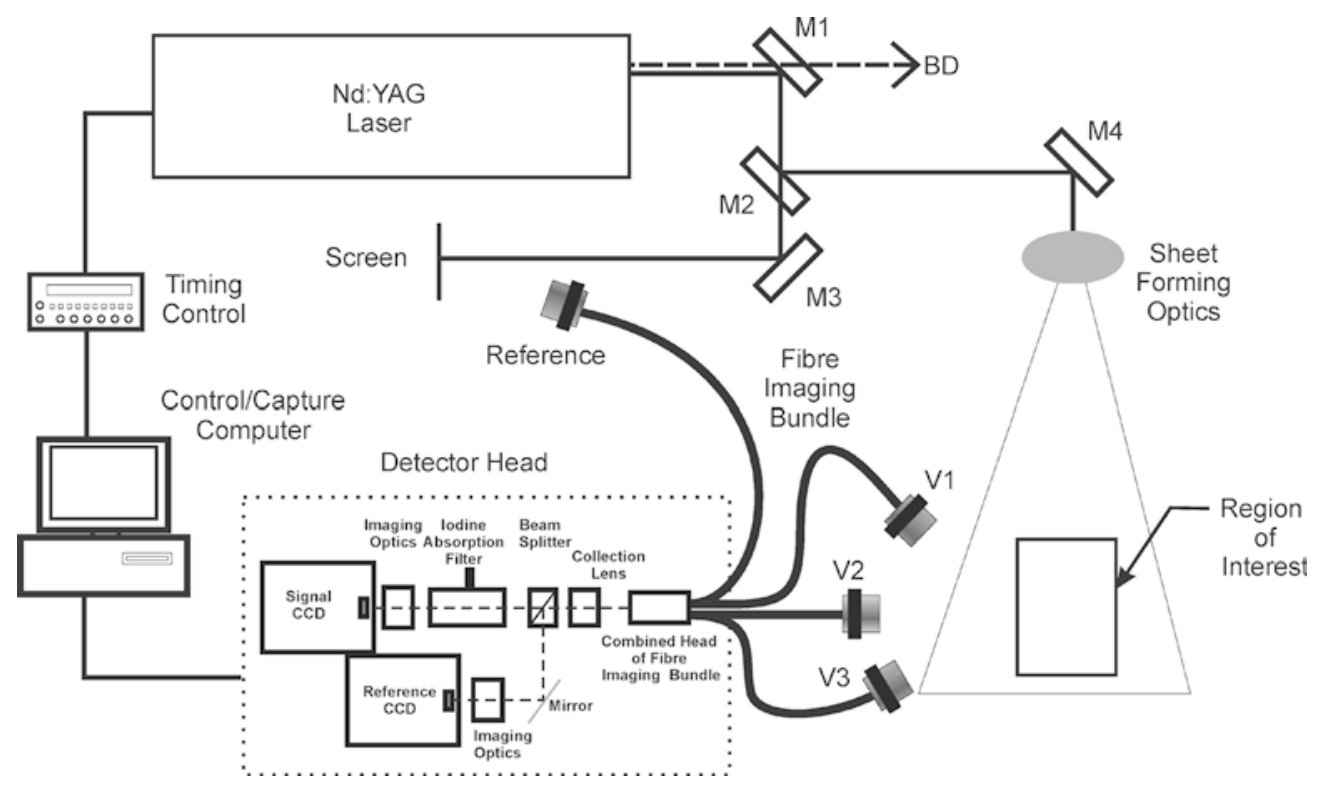

Fig. 3. Experimental set-up. A schematic of the experimental arrangement for the PDV experiment. $-532 \mathrm{~nm}$ beam; $-1064 \mathrm{~nm}$ beam; M1 and M2 $532 \mathrm{~nm}$ dicroic mirrors; M3 and M4 turning mirrors, BD-1064 $\mathrm{nm}$ beam dump 
temperature set so that all of the iodine present has sublimed to vapour. A PID controller controls the temperature of the oven $\pm 1{ }^{\circ} \mathrm{C}$. This form of iodine cell is termed a starved cell. A schematic of the detector head is shown as a sub-figure in Fig. 4. Note this has the same basic layout of 4 cameras and iodine cell as the single component head shown in Fig. 2a. The image transfer optics port an image into the head system that is first split into two paths with a $50 / 50$ non-polarising beam splitter. One path is directed to the reference camera via a turning mirror, this corrects the orientation of the image, while the second is directed through the iodine cell to the signal camera.

The image coupled into the detector head is delivered to it by an imaging fibre bundle. This is a coherent array of fibres that is split into four channels and each channel is made up of $500 \times 600$ fibres and is each $4 \mathrm{~m}$ long. Three of these are used to view the same region of interest from three different directions. The fourth, as shown in Fig. 3, images a small percentage of the laser pulse that has passed through a $532 \mathrm{~nm}$ dichroic mirror and scattered off a screen. This provides a means of monitoring the frequency of each laser pulse. To demonstrate the combining of channels an example image from the signal camera of the combined three views and the laser beam reference view is shown in Fig. 4. The top left and bottom right and left are the three views of a reference target of crosses and views an area $200 \times 200 \mathrm{~mm}$. The perspective distortion of the target highlights the different directions of the three views. The top right corner of the image in Fig. 4 is the fourth channel used to image the laser beam and does not view the reference target.

The use of the imaging fibre bundle reduces the total number of cameras used to two. The inclusion of the referencing channel allows the system to use only a single iodine cell and hence only a single calibration of the cell is required. Imaging the pulse for frequency referencing also allows the distribution of laser power in the pulse to be recorded as well as the frequency distribution across the

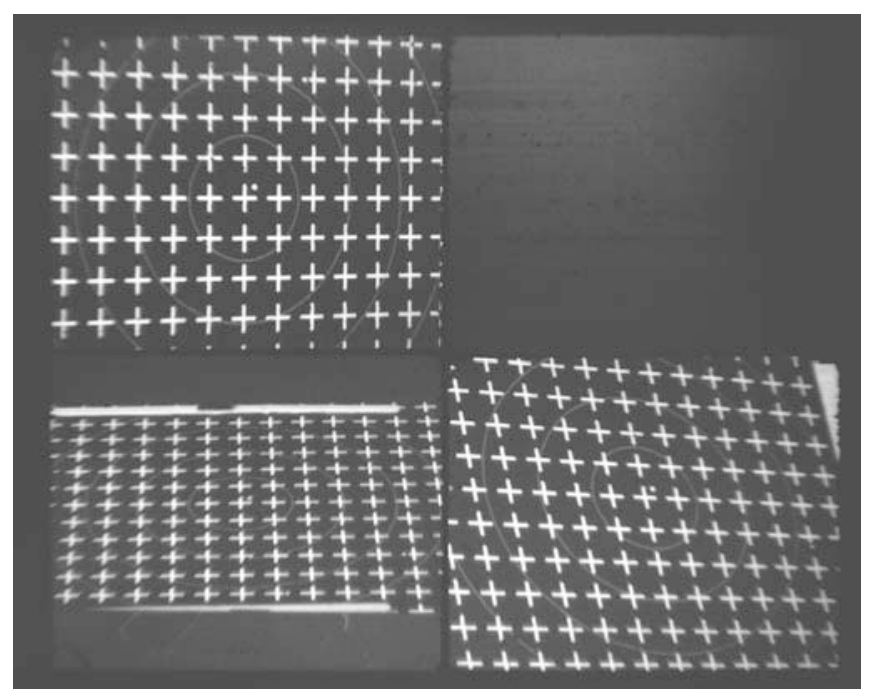

Fig. 4. Image of the target. An example image of the view through the image bundle of a reference target captured with only one camera. The area viewed of the target is $200 \times 200 \mathrm{~mm}$ beam wave front. This PDV system significantly reduces the number of components and the complexity compared to systems reported in the literature.

Processing procedure

Camera control, image capture and processing for the PDV system have been integrated into a single software bundle that is based on the camera's control macro language (LaVision DaVis 6.0). The macro language allows for the capture, processing and display of data as well as a facility to port data to $\mathrm{C} / \mathrm{C}++$ DLL functions for specific and fast processing. Several functions included in the software are incorporated into the processing of the PDV data.

The use of the imaging fibre bundle to capture all three views onto a single image has introduced extra processing of the collected images compared to a system that has three completely separate heads for each view. As part of the collection process, the two raw images from the signal and reference cameras have had a background subtracted. This background is the noise accumulated in the exposure as well as the read out noise. Before proceeding with processing the data to velocity vectors, each separate view is extracted from the raw images into separate buffers. These buffers are then de-warped and the data interpolated and mapped onto a regular grid. The de-warping coefficients are first defined using a target image of crosses as shown in Fig. 4. The software searches through the images separately locating the centre of the crosses using a correlation method. In determining the de-warping coefficients, the location of the crosses is first mapped to a normalised space of 1 to 1 across the image and bottom to top of the image. The de-warping coefficients are then determined from curve fitting a third order polynomial. The data is then mapped onto a defined grid space with a calculated scale. The coefficients are stored for this specific view. Of the six views extracted from the signal and reference images, three from the signal and three from the reference image, a master view is selected and initially dewarped. All other views are first mapped to the normalised space and then mapped onto this master so that each view has the same origin within the image and each pixel has the same scale.

De-warping of the views corrects the images for distortion. There are several distortions that are present. There is a general perspective distortion in each view, which is a function of the viewing direction. The imaging system can introduce barrelling or pincushion distortion. While these distortions are relevant for both cameras, there is a third distortion that is also present between the cameras. This is due to misalignment of the cameras and for any change in the magnification of the image after the beam splitter onto the image array of the cameras. Hence six separate sets of de-warping coefficients are used.

Once the data has been de-warped, interpolated and mapped it can be passed into a processing procedure to determine velocities. Before division of the signal and reference images, the data is corrected using a 'white card' correction and put through a low pass filter. The white card correction corrects the data for variations of signal sensitivity in the CCD and vignetting of the signal across 
the array. A low pass filter can be used to reduce the effects of laser speckle. After the division of each of the three views the intensity ratio is converted to frequency. This conversion is via a polynomial fit of the iodine transfer function. The transfer function of the absorption band used is measured by frequency tuning the laser across the range of the absorption band. This data is then fitted to a model of the absorption spectra using the code developed by Forkey (1996). After the conversion, the data at each point in the view is an absolute measurement of the scattered laser frequency. A measurement of laser frequency for the measurement pulse is determined using the same fitting procedure using the image of the laser pulse captured by the fourth leg of the fibre-imaging bundle. The Doppler difference is then calculated using Eq. (1) and the velocity magnitude of the component defined by the laser and viewing direction can be calculated. This procedure is repeated for each of the three views. The data can then be mapped onto a regular orthogonal grid generating the three orthogonal components of the measured velocity over a plane in the reference frame of the experiment.

\section{5}

\section{Results}

\section{1}

\section{The velocity field of a rotating disk}

The system has been developed with a specific aim to measure velocities of a flow using particles seeded into the flow as the scattering medium. Any moving object however will Doppler shift a light source directed at it. In the development stage of the system a rotating disk has been used to provide a moving field of a well-defined velocity. Results of the system are presented here using a rotating disk $200 \mathrm{~mm}$ in diameter that has been coated with flat white paint with the aim to give near uniform reflection. The disk is illuminated by the laser at an angle that is near perpendicular to the rotational axis. The three views are arranged so that all three are sensitive to the rotating field. Instantaneous results of the velocity field were collected using the pulsed Nd:YAG described earlier as the light source.

The computed velocity field for the three different views of the disk are shown in Fig. 5. The results have been smoothed with several passes of a low pass filter to reduce the effect of speckle noise. The residual effects of laser speckle are however still evident in the image as well as the noticeable fluctuations over larger areas. This can be linked to changes in the distributions of the laser power across the wave front. A significant proportion of this effect is removed using the white card correction for laser power distribution however there is a small amount of AC fluctuation still present.

The vector map of the velocity field shown in Fig. 6 has been generated by mapping the scalar field of the three views in each data set onto a regular orthogonal grid. The transformation matrix is generated using the velocity maps and information of the view directions. In the vector map, only every tenth vector has been shown. Noise present in the instantaneous measurement results in a vector field that has some bad vector directions. However,
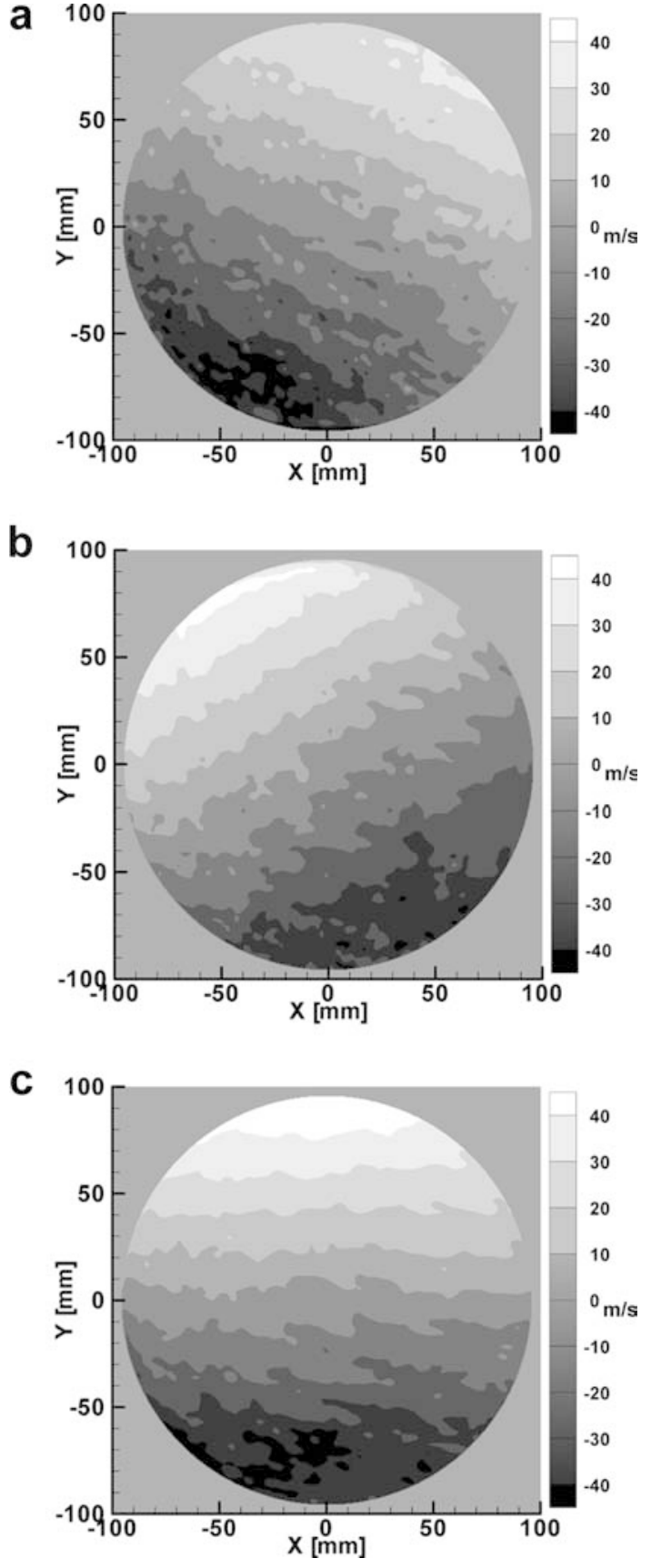

Fig. 5. Computed velocities from each view for the YAG. The computed instantaneous velocity field for the different views using a pulsed frequency doubled Nd:YAG laser

generally the velocity field is representative of the velocity field that can be expected of the rotating disk.

\section{2 \\ The instantaneous velocity field of a jet}

The PDV has also been used to measure the instantaneous velocity field of a jet flow that has been seeded uniformly with particles. The jet exits a $70-\mathrm{mm}$ diameter pipe nozzle that had a bulk exit velocity of $30 \mathrm{~m} / \mathrm{s}$. Particles of a mean diameter of $0.6 \mu \mathrm{m}$ were introduced into the jet flow well up stream from the nozzle exit. A raw image from the reference camera of the flow is shown in Fig. 7. This figure shows the three views of the jet and a single view of the 


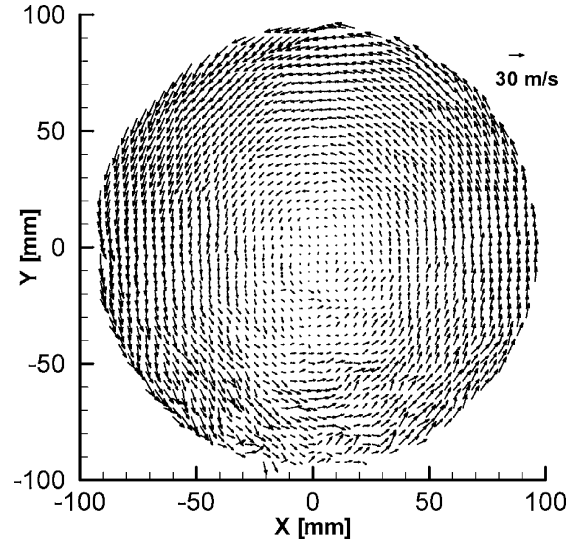

Fig. 6. Vector field of the rotating wheel. The processed velocity vector fields of the rotating disk

intensity of the laser pulse in the top right hand corner of the image. Poor alignment of optics within the laser is the most likely reason for variations in this intensity image of the laser pulse. This raw image shows the effect of laser speckle on the scattered signal. As described earlier, the speckle pattern is removed using a low pass filter. This image also shows that for this measurement the PDV system was set-up in a different configuration. The views on the left of the image have been taken from the same side of the laser light sheet. The three third view (bottom right) is a mirror reflection of the bottom left view. This can be seen in the shape and positions of structures in the seeded jet. The full width of the jet has been imaged and regions where the non-seeded ambient fluid is present are evident by the lack of signal. For the calculation of velocity vectors, only the region marked by the rectangle has been used because no results will be computed where there is no signal. Also in this region the scattered signal to the reference camera is near uniform.

The computed and mapped orthogonal field for the marked region of Fig. 7 is shown in Fig. 8a. Vectors indicate the direction and magnitude of the in-plane components of velocity while the colour map indicates the magnitude of the out-of-plane component. Only one in eight velocity vectors are shown. This velocity field is typical of what can be expected in the near field of the jet. The region marked in Fig. 8a shows the presence of reverse flow. This region is magnified in Fig. $8 \mathrm{~b}$ where all computed velocity vectors have been shown. The vector map clearly shows the presence of a vortex whose axis at the time of capture is pointing outof-plane. This instantaneous vector field is smooth and has a general bulk velocity similar to the bulk flow velocity of the jet.

\section{3}

\section{Errors}

There are several areas within which errors to the calculation of a velocity vector using the PDV technique can be associated. The experiments investigating the velocity field of the rotating disk have highlighted the following sources of errors as potentially the most significant in the current technique.

Image registration-this is the accuracy of the registration between the signal and reference images. Accurate registration of the two images in principle would remove

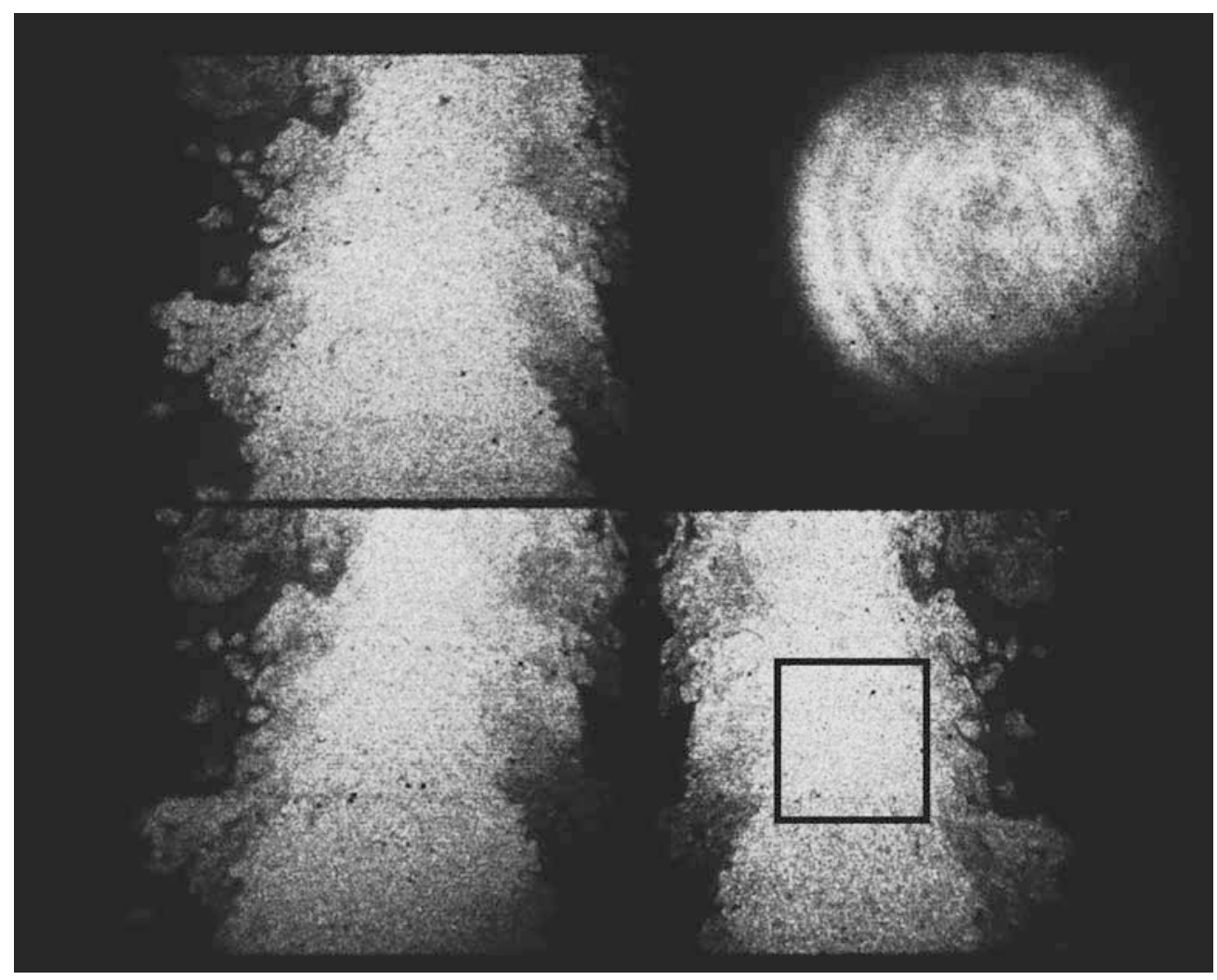

Fig. 7. Raw image view of the jet (square showing region of interest). A raw image from the reference camera of scattered signal from the seeded jet 

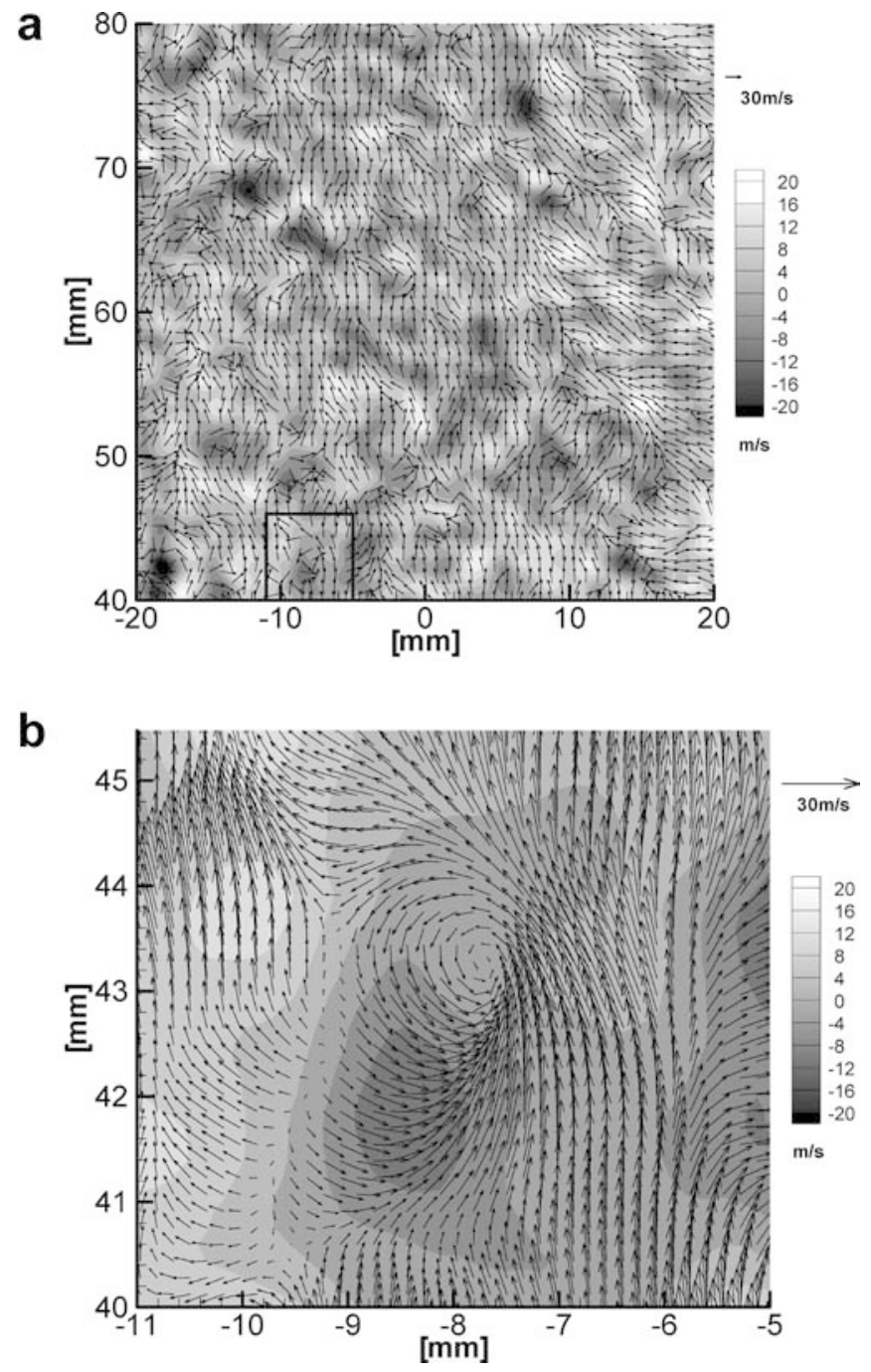

Fig. 8a, b. Velocity field of the jet. a Large view; b zoomed view. The computed orthogonal velocity field of the jet: a for the region shown in Fig. $7 ; \mathbf{b}$ for the region shown in $\mathbf{a}$

the noise introduced by laser speckle. However, if there is some error in the registration, an error would be introduced after the division that would appear similar to speckle noise.

Modelling of the iodine transfer function-the shape of the transfer function is critically dependent on the vapour pressure of Iodine in the cell and hence the operating temperature of the oven. As highlighted by other authors, the use of a starved cell will minimise these effects. Accurate modelling of the shape of the transfer function is however needed and assumptions of linearity or Gaussian shape of the function over the region of the measurement can led to significant errors.

Measurement of laser frequency-the pulsed laser has a significant pulse to pulse frequency fluctuation that requires individual measurement of each measurement pulse frequency to correct each data point.

Image registration between views-each view shown in Figs. 7 and 8 has been mapped onto a single scale for that data set. The accuracy of how well these individual views register with each other will affect the result of the matrix mapping to the orthogonal representation of the data.

6

\section{Conclusions}

A PDV technique has been described that is capable of measuring the three instantaneous components of velocity over a two-dimensional region. All three views needed to define a three-dimensional velocity field have been recorded using a single pair of signal and reference cameras by porting the three views through a fibre-imaging bundle. The legs of this bundle combine into a single array that is imaged through the PDV detection head. Laser frequency stability is monitored and measured using the fourth leg of the bundle allowing for correction for any frequency movement of the laser for each instantaneous measurement. The use of a single PDV detector head minimises the number of CCD array cameras that are used to a total of two and only a single iodine cell is used for both measurement of the three velocity components and reference measurement of the laser frequency. Only a single frequency scan is needed to define the transfer function of the iodine cell for converting measured intensities to frequency. Any drift in the iodine cell characteristics is then constant across all measured components. The innovations of the current set-up of PDV will allow for simple integration of the technique into experimental rigs.

\section{References}

Chan VSS, Heyes AL, Robinson DI, Turner JT (1995) Iodine absorption filters for Doppler global velocimetry. Meas Sci Technol 6:784-794

Elliot GS, Beutner TJ (1999) Molecular filter based planar Doppler velocimetry. Prog Aero Sci 35:799-845

Ford HD, Tatam RP (1997) Development of extended field Doppler velocimetry for turbomachinery applications. Optics Lasers Eng 27:675-696

Forkey JN (1996) Development and demonstration of filtered Rayleigh scattering - a laser based flow diagnostic for planar measurements of velocity, temperature, and pressure. Final Technical Report for NASA Graduate Student Researcher, Fellowship Grant \#NGT50826, Princeton University

Komine H (1990) System for measuring velocity field of fluid flow utilizing a laser-Doppler spectral image converter. US Pat No. $4,919,536$

Meyers JF (1991) Generation of particles and seeding. Von Karman Institute Lecture Series 1991-08. Available from NASA Report Server

Meyers JF, Fleming GA, Althoff Gorton S, Berry JD (1998) Instantaneous Doppler global velocimetry measurements of a rotor wake: lessons learned. In Proceedings of the Ninth International Symposium on Applications of laser techniques to fluid mechanics, Lisbon, Portugal, 13-16 July

Meyers JF, Komine H (1991) Doppler global velocimetry: a new way to look at velocity. Laser Anemometry 1:289-296

Miles RB, Yalin AP, Tang Z, Zaidi SH, Forkey JN (2001) Flow field imaging through sharp-edged atomic and molecular "notch" filters. Meas Sci Technol 12:442-451

Mosedale AD, Elliott GS, Carter CD, Beutner TJ (2000) Planar Doppler velocimetry in a large-scale facility. AIAA J 38:1010-1024

Nobes DS, Ford HD, Tatam RP (2002) Three dimensional planar Doppler velocimetry using imaging fiber bundles. In Fortieth AIAA Aerospace Sciences Meeting and Exhibit, Reno, Nevada, 14-17 January 2002. AIAA, Reston, Va. 
Reinath MS (1997) Doppler global velocimeter development for the large wind tunnels at Ames Research Center. NASA TM-112210

Roehle I (1996) Three-dimensional Doppler global velocimetry in the flow of a fuel spray nozzle and in the wake region of a car. Flow Meas Instrum 7:287-294

Roehle I, Schodl R, Voigt P, Willert C (2000) Recent developments and applications of quantitative laser light sheet measuring techniques in turbomachinery components. Meas Sci Technol 11:1023-1035
Samimy M, Wernet MP (2000) Review of planar multiple-component velocimetry in high speed flows. AIAA J 38:553-574

Smith M, Northam G, Drummond P (1996) Application of absorption filter planar velocimetry to sonic and supersonic jets. AIAA J 34:434-441

Tropea C (ed) (2001) Special feature: Molecular filter based diagnostics. Meas Sci Technol vol 12 